\title{
Nicaragua, enfoque estratégico de las PYMEs en un mundo globalizado
}

Sandra Cuadra ${ }^{1}$

1 Maestría en Administración y Dirección de Empresas (MADE) Facultad de Ciencias Económicas y Empresariales de la UCA. Apdo. 69, Managua, Nicaragua.

\section{Recibido: abril 2006 / Aceptado: mayo 2006}

ESTA INVESTIGACIÒN ABORDA LA SITUACIÒN DE LA PEQUEÑA y mediana empresa (PYME) nicaragüense desde una perspectiva nueva en Nicaragua: su enfoque estratégico respecto al proceso de globalización. Las PYMEs son importantes para el desarrollo económico y social de Nicaragua por su contribución al Producto Interno Bruto (PIB) y al empleo: contribuyen con el $32.6 \%$ del PIB nacional y se estima que ocupan entre el 40 y 50\% de la población económicamente activa. Partiendo de experiencias exitosas en otros países, este trabajo propone líneas de acción estratégicas y desafíos, señalando las condiciones para el desarrollo; también plantea el rol gubernamental para la implementación del marco legal normativo y programas y proyectos que fortalezcan las PYMEs, reducción de brechas tecnológicas, cadenas de asociatividad productiva, desarrollo de zonas francas, constitución de redes empresariales, fomento de clusters competitivos y formación de alianzas estratégicas.

Palabras clave: pequeña y mediana empresa-Nicaragua, planificación empresarial, competencia económica, Nicaragua-política económica

\section{Introducción}

La globalización presenta para las PYMEs un nuevo escenario donde es necesario considerar la dimensión internacional como factor determinante de estrategias y políticas. El mundo se está convirtiendo en una "aldea global" donde la información al instante aumenta la velocidad de las actividades económicas. En este ámbito, las PYMEs siguen siendo parte importante de la economía de cualquier país: las PYMEs representan casi el 99\% de las empresas, emplean a más del 55\% de la fuerza laboral y generan más del 40\% del PIB mundial

En Nicaragua, el Instituto Nicaragüense de Apoyo a la Pequeña y Mediana Empresa (INPYME) definealasPYMEs “comoempresas formales ${ }^{1}$ manufactureras, agroindustriales, comerciales y de servicios, con mas de dos trabajadores y menos de cien, con potencial de desarrollo en un ambiente competitivo". Esta definición excluye de las PYMEs a las 
empresas agropecuarias.

En Nicaragua, las PYMEs son consideradas el principal promotor del desarrollo económico, porque son la mayoría de las empresas nicaragüenses, entre el 90 y 95\%, con un aporte del 90\% del empleo, y son el principal instrumento para combatir la pobreza. Su valor agregado, mayor del 50\% en los establecimientos del sector urbano, se traducen en una contribución de $32.6 \%$ del PIB.

Además de estas características, las PYMEs:

- Generan empleo y utilizan mano de obra y materia prima nacionales, generando efectos multiplicadores.

- Requieren poca inversión y financiamiento.

- Son flexibles para orientarse a la moda y a las necesidades cambiantes de los consumidores, con capacidad para atender a nichos de mercado y segmentos de clientes que buscan atención personalizada.

- Rescatan las habilidades, el saber y la tecnología tradicionales.

- Contribuyen a la diversificación geográfica de la industria.

- Evitan los monopolios.

- Generan industrias apropiadas para el mercado que abastece.

- Participan activamente en el crecimiento de las exportaciones nacionales, distribución del ingreso, balanza de pagos, acumulación de capital y productividad, y contribuyen a la autosuficiencia del país.

- Emplean a personas discapacitadas, mayores o con dificultades.

- Utilizan generalmente tecnología intermedia, adaptada a la oferta de trabajo.

En Nicaragua, el Ministerio de Fomento, Industria y Comercio (MIFIC) tiene la misión de "Impulsar la productividad, eficiencia y competitividad de cadenas y enjambres intersectoriales, la industria y otros sectores no agropecuarios, apoyándose en el desarrollo, transferencia de la tecnología y la capacitación gerencial con énfasis en la pequeña y mediana empresa" para lo que ha creado la Dirección de Políticas y Fomentos a las PYMEs (DPYME). Por su parte, el Instituto de la Pequeña y Mediana Empresa (INPYME) ha diseñado la "Propuesta de Desarrollo para MIPYME". En Centroamérica hay un proyecto de apoyo a la micro y a la pequeña empresa (PROMICRO $)^{2}$ que promueve el fortalecimiento y la promoción de organizaciones gremiales.

\section{Problemas de las PYMEs}

Los pequeños y medianos empresarios enfrentan problemas en gestión, información, calidad, mercadeo, acceso a financiamientos y tecnología, entre otras.

En cuanto a la gestión, el empresario propietario tiene que atender a tantos detalles administrativos, financieros y productivos que se le dificulta el diseño de estrategias a largo plazo. Este problema se acentúa cuando las relaciones empresariales son informales, pues pueden estar mal informados sobre su propia estrategia financiera y su potencial productivo. Este desconocimiento de los instrumentos de gestión empresarial les dificulta la toma de decisiones económicamente viables a su desarrollo. Las PYMEs usualmente no cuentan con registros adecuados que sirvan como herramientas en la toma de decisiones. 
Los recursos humanos no tienen las competencias, destrezas y habilidades que demanda el mercado, pues su formación es empírica; los salarios suelen ser desalentadoramente bajos. Las PYMEs absorben la mayoría de la fuerza laboral que todos los años entra al mercado del trabajo, creando empleos generalmente poco tecnificados, poco calificados, poco productivos y poco remunerados.

En cuanto al mercadeo, las PYMEs tienen dificultades para exportar, lo que obstaculiza el proceso de internacionalización, que exige abordar individualmente las tareas de producción, comercialización y logística de exportación. Esto muchas veces está fuera de su alcance por limitaciones financieras, de personal, infraestructura organizativa, conocimientos de los mercados y destrezas.

Otro problema es la escasez de capital con que inician y permanecen las PYMEs, que dificulta el acceso a créditos, porque los bancos las ven de alto riesgo. Si una PYME contara con una relación normal de capital propio versus endeudamiento, en un contexto económico estable y el costo del crédito inferior al 20\%, seguiría en riesgo de desinversión, salvo que su utilidad supere el $12 \%$ sobre el activo.

En cuanto a la tecnología, la capacidad de las PYMEs para satisfacer a sus clientes suele ser limitada pues carecen de instrumentos para mejorar los productos, su imagen comercial, su poder de negociación frente a clientes y proveedores, y los trabajadores están poco articulados para solucionar problemas, tomar decisiones y planificar el trabajo. Pese a todo, los problemas de la PYMEs no se deben a su tamaño, sino a su aislamiento de contextos políticos, institucionales, económicos y territoriales de apoyo.

\section{Lo que puede hacer el gobierno}

Según la Constitución Política de Nicaragua, el estado debe promover el desarrollo económico del país. Durante 2001, el gobierno elaboró tres documentos relacionados con las PYMEs: "Memoria del I Congreso Nacional PYME" elaborado por la Dirección de Políticas de Fomento a las PYMEs (DPYME-MIFIC), de marzo; "Plan Estratégico" del Instituto Nicaragüense de Apoyo a la Pequeña y Mediana Empresa (INPYME), de mayo; y "Propuesta de Desarrollo para la Micro, Pequeña y Mediana Empresa Nicaragüense”, de junio.

Estos documentos crean una marco institucional para el desarrollo de las PYMEs, lo que representa un salto cualitativo para enfrentar la apertura del mercado y la fuerte competencia que enfrenta la economía nicaragüense, en tanto este marco legal no forme parte únicamente de una realidad imaginaria. Por tanto, el gobierno debe aplicar este marco legal en acciones concretas.

Sin embargo, los documentos por sí mismos, muestran un conflicto de poderes y contradicciones, en tanto en los documentos se enfatiza la división de responsabilidades de cada instancia desde una visión jerárquica y no de convergencia de acciones enfocadas al desarrollo, lo que de profundizarse, podría ser una barrera en el alcance de las metas propuestas y limitar, en lugar de facilitar el crecimiento y fortalecimiento de las PYMEs. 


\section{Impacto de la Globalización en las PYMEs}

El Fondo Monetario Internacional (FMI), define la globalización como "la interdependencia económica creciente en el conjunto de los países del mundo, provocada por el aumento del volumen y de la variedad de las transacciones transfronterizas de bienes y servicios, así como de los flujos internacionales de capitales, al mismo tiempo que por la difusión acelerada y generalizada de la tecnología”. En esta visión hay dos conceptos clave: interdependencia y quedarse en la manifestación del fenómeno sin interesarse por sus actores políticos y económicos. La globalización es un proceso dirigido por los grandes países del norte, y los países pobres sólo lo pueden absorber con intervención del estado.

Las oportunidades brindadas por la globalización están dadas por:

- Posibilidades de progreso en organización, eficacia, productividad, difusión de conocimientos, mejoras del nivel de vida y acercamiento entre los hombres.

- Oportunidad de accesar a mercados anteriormente fragmentados, originada por la formación de alianzas y tratados de libre comercio.

- Los flujos de información, tecnología y capital de cartera han aumentado su movilidad para economías con poca capacidad de generación endógena.

- Disminución de costos directos e indirectos.

- Aumenta la competencia pues permite a las PYMEs buscar mayor eficiencia, mejor calidad, mejorar sus procesos y precios competitivos.

- Contribuye a la desarticulación de los oligopolios.

Las amenazas generadas consisten en:

- Las fallas de mercado que genera la globalización económica y deficiente entorno institucional en el que operan.

- El ingreso de nuevos productos al mercado nicaragüense por los acuerdos de libre comercio y eliminación de barreras arancelarias.

- El rápido aumento de los salarios.

- La declinación del empleo en la industria manufacturera.

- Las empresas locales no suelen estar preparadas para enfrentar la competitividad, la comercialización y la productividad de las transnacionales.

- El estancamiento tecnológico.

\section{Efectos de la Globalización en las PYMEs}

La reducción de barreras arancelarias ha introducido el consumo masivo. Esta reducción beneficia a los importadores, pero afecta a los productores locales de productos y materias primas, que no pueden competir con precios más bajos.

La disponibilidad de una gran cantidad de productos, insumos, componentes y servicios de bajo costo provenientes del exterior afecta y tensiona los sistemas productivos de muchos países subdesarrollados, donde la rápida apertura económica se ha traducido en desarticulación de las cadenas productivas y ensanchamiento de la brecha de competitividad y productividad entre el sector moderno, constituido por un reducido número de empresas grandes, que concentran un fuerte poder económico, y un gran número de PYMEs. 
Se han creado grandes corporaciones en ciertos sectores industriales, a las que se han vendido las PYMEs sectoriales: a través de una ola de expansiones, fusiones y adquisiciones, el poder económico se ha concentrado en mega empresas con sede en países desarrollados, con serias implicaciones para el sistema de competencia global, para la generación de oportunidades para las empresas de menor tamaño y la creación de empleo.

Sin embargo, hay PYMEs que han visto en este proceso de globalización una oportunidad para reforzarse y expandirse. Son las ubicadas en territorios caracterizados por vínculos de cooperación inter-empresarial, de colaboración entre el sector público y privado y de una intensa relación entre instituciones de servicio y el sector productivo. Son PYMEs inmersas en sistemas productivos integrados, fincados a nivel local y caracterizados por un estrecho entramado entre las empresas y entre éstas y otros actores locales, como instituciones públicas y privadas de apoyo financiero, de formación, investigación, asistencia técnica y de servicio. El desarrollo y enfoque de actividades hacia la protección del medio ambiente está siendo considerado como vital para la economía globalizada.

Es necesario realizar una profunda reflexión alrededor de las PYMEs sobre dos alternativas: a) ¿tienden a desaparecer con la globalización? b) ¿se suman, se adaptan y compiten en el proceso de la globalización?

\section{Enfoque estratégico de las PYMEs frente a la Globalización}

La supervivencia y evolución de una empresa dependen de su capacidad de crear y mantener ventajas competitivas en sus mercados. Por ello, se define estrategia como un proceso continuo para identificar aspectos en los que la empresa puede ser más eficiente que la competencia, utilizando menos recursos para obtener el producto o prestar el servicio, para desarrollar una oferta con valor único.

Para analizar la estrategia que deben seguir las PYMEs, es necesario analizar su situación actual; a continuación, hay que definir cuál se quiere que sea su situación en el futuro y lograr que esa aspiración sea el motor principal de la actividad. No se trata de hacer análisis complicados, sino de generar una motivación capaz de anticipar el futuro incluyendo una imagen viva y evolutiva de dónde queremos estar en 5 o 10 años. Esa motivación creada por el empresario tiene que ser lo suficientemente atractiva como para que sea compartida por el resto del personal, para que lo convenza y lo entusiasme tanto como a su creador, se comprometa con ella y se constituya en la guía del trabajo diario.

Es importante que todos los actores concuerden en una línea de pensamiento estratégico orientado al desarrollo: empresarios, gobierno, cámaras y gremios, universidades e instituciones financieras. No basta el financiamiento, el desarrollo tecnológico ni la capacitación, pues se trata de procesos ligados, cambios estructurales de política económica nacional de estabilización y de ahorro interno.

\section{Líneas estratégicas a seguir}

El proceso convencional de visión estratégica no es útil para las PYMEs. En su lugar, 
adquiere fuerza la intuición del empresario que, muchas veces, rompe los moldes y que había sido casi anulada por la planificación estructurada. Pero tampoco se pude dejar todo a la intuición obviando la planificación. Mientras mayor es la incertidumbre, mayor es la necesidad de planificación.

Es necesario fijar los objetivos, estrategias y acciones necesarias para conseguirlos. Para desarrollar un Plan Estratégico, las PYMEs deben utilizar la herramienta conocida como análisis FODA (fortalezas, oportunidades, debilidades y amenazas). En definitiva, se trata de conocer cual es la posición estratégica y valorar la actuación de las empresas.

\section{A continuación:}

1. Definir claramente el objetivo fundamental de la empresa.

2. Desarrollar metas comprobables por área, con fechas para alcanzarlas.

3. Desarrollar estrategias acordes a las metas, determinar cómo se implementarán y definir los recursos humanos y financieros necesarios.

4. Diseñar el plan operativo incorporando los presupuestos.

5. Ejecutarlo y supervisarlo.

Las acciones deberán ser corregidas cuando sea necesario, sin olvidar que la flexibilidad es el factor más importante para alcanzar los resultados esperados, pues en el entorno actual no existe la mejor opción. Hay que ir haciendo camino al andar, por lo que el plan, continuamente retroalimentado, debe ser flexible para admitir las estrategias emergentes que se percibanEstaba y estar abierto a descubrirlas.

\section{La gestión de los recursos humanos}

Las PYMEs tienen dificultad para contratar y retener personal calificado para sus operaciones. Hay algunos métodos de gestión de recursos humanos que se adaptan a las PYMEs que no requieren de grandes inversiones:

- La gerencia puede ser enfocada de forma que permita crear un ambiente más participativo, comunicativo y de cooperación mutua.

- Realizar reuniones de discusión participativa, para discutir los problemas de la empresa para buscar posibles soluciones.

- Incorporar sistemas de auto evaluación.

- Informar a todo el personal respecto a: plan operativo de la empresa, perspectivas, metas especiales y situación económica.

- Transmitir a los empleados que ellos también son socios de la empresa, delegando responsabilidades y asignando tareas de confianza.

- La formalización y documentación de contratos de personal, manual de administración, manual de funciones.

- Formalización y documentación de manual de terreno, donde se indique detalladamente los pasos en cada etapa del proceso productivo.

- Promover la asociatividad como mecanismo para la superación de carencias individuales. 
de recursos humanos que han mostrado eficacia en las grandes organizaciones. Uno de los principios sobre el que se apoya parte de la eficacia de las PYMEs es la coherencia entre filosofía, estrategia, sistemas, estilos, valores y acciones de la organización. Las PYMEs requieren especialmente de una incorporación metodológica coherente y progresiva para desarrollar sus estructuras y llevar a cabo los nuevos objetivos en recursos humanos. La utilización aislada de ciertas técnicas en materia personal produce, inevitablemente, resultados empobrecidos.

Cuando se afirma que la empresa debe perder rigidez y adquirir una mayor flexibilidad organizativa, se tiende sólo a cambiar las estructuras. Pero es preciso ver el problema en el conjunto de sus implicaciones, con los requerimientos de cambios de actitudes, estilos de dirección y control consecuentes; con las nuevas demandas de información y comunicación, y la necesidad de reorientar las políticas retributivas y de apreciación del desempeño. Esta visión integrada de los sistemas de gestión permite a la empresa desarrollar su propia filosofía y estilo de dirección y las intenciones estratégicas se realizan en las acciones.

La política de capacitación y actualización de los recursos humanos requiere de un permanente proceso de capacitación para adecuarlos a las nuevas tecnologías operativas y organizacionales. La capacitación del personal de los niveles de conducción y operativo es importante debido a los nuevos problemas que plantean los cambios en la organización productiva a nivel regional. Para redefinir el perfil del nuevo trabajador en el contexto actual, la capacitación no debe centrarse sólo en los aspectos técnicos; también sobre el proceso productivo como una totalidad, para integrarlo a su práctica laboral.

\section{La tecnología como herramienta generadora de capacidad}

El concepto de tecnología ha evolucionado, y ya no sólo se refiere a máquinas y equipos, sino a todo el know-how, información, conocimientos y decisiones necesarias para mantener una empresa competitiva en el largo plazo. Por tanto, del concepto cuasi-estático de transferencia de tecnología, se está pasando al concepto dinámico de gestión estratégica de tecnología, donde la tecnología se constituye en un elemento estratégico en el proceso de toma de decisiones.

La gestión de tecnología e innovación organizacional son elementos clave en la dirección. Corrientes culturales empresariales de excelencia que incorporan nuevos valores, promueven el trabajo en equipo y círculos de calidad, que rompen con los modelos excesivamente jerarquizados en la toma de decisiones. Por tanto, se revalora el rol del factor humano como causa y objeto del desarrollo y una mayor valorización de la creatividad y la capacidad de innovar y emprender.

La gestión de intangibles (tecnología e información) se ha convertido en el factor decisivo de la competitividad empresarial desde la doble perspectiva externa e interna de la empresa:

a) A lo externo, la rapidez del cambio tecnológico y los costos de Investigación y Desarrollo hacen necesario que las PYMEs estén informadas sobre las principales actividades de sus competidores y clientes. Muchas empresas han desaparecido por aplicar ineficientemente nuevas tecnologías, retrasando la comercialización de productos 
y perdiendo oportunidades, por no identificar adecuadamente las necesidades de los clientes.

b) A lo interno, es necesario identificar los puntos críticos del proceso de innovación, para hacer llegar la información a las áreas que la necesitan para desarrollar con eficiencia las distintas fases del proceso tecnológico (investigación, desarrollo, diseño, fabricación, comercialización).

En la última década, hay una eclosión de técnicas de gestión empresarial con un objetivo común: "hacer más con menos". Se persigue reducir la fuerza de trabajo, acelerar los procesos de diseño y lanzamiento comercial de nuevos productos, acortar los plazos de fabricación o aumentar la variedad de la gama de productos.

El conjunto de estas transformaciones tiene implicaciones relevantes: por un lado, se dirigen a incrementar la productividad empresarial que se refleja en una disminución del tiempo necesario para culminar la innovación desde las fases iniciales del proceso productivo hasta su comercialización, o en el número de horas necesarias para producir el bien o servicio. Con esto se ha demostrado que, aunque se destruye empleo a corto plazo, se asegura la supervivencia de la empresa a medio y largo plazo en un entorno altamente competitivo, contribuyendo a consolidar los restantes empleos; y se contribuye al crecimiento de la organización, permitiendo la eventual creación de empleo a largo plazo. Por otro lado, este conjunto de innovaciones facilitan la aparición de nuevos sectores de actividad económica que eran desconocidos hace tan sólo una década.

El comercio tecnológico brinda acceso a información, comunicación, demostración, mercadeo y comercialización; por tanto, debe explotarse para mejorar los beneficios. El estado, como facilitador, debe propiciar la comunicación virtual y facilitar a las PYMEs páginas de comercio electrónico para entrar al mercado internacional. El control de factores externos como el control de calidad, el respeto a los derechos de autor y la protección a la propiedad intelectual es clave para un ambiente confiable al desarrollo y la creación de innovaciones.

\section{La asociatividad entre PYMEs}

La asociatividad es "un mecanismo de cooperación entre PYMEs, donde cada participante, manteniendo su independencia jurídica y autonomía gerencial, participa voluntariamente en un esfuerzo conjunto para la búsqueda de un objetivo común”3.

Estos objetivos pueden ser coyunturales: adquisición de materia prima; o generar una relación más estable: investigación y desarrollo de tecnologías para beneficio común. El esfuerzo conjunto de los participantes en la asociatividad puede materializarse de distintas formas, desde la contratación de un agente de compras o ventas pagado conjuntamente, hasta la formación de una empresa con personería jurídica y patrimonio propio.

Las PYMEs, en diferentes países y bajo diversas modalidades, han experimentado e instrumentado varios mecanismos para enfrentar conjuntamente, determinadas amenazas o superar debilidades específicas y comunes. La globalización económica está redefiniendo los procesos de manufactura al localizar las fábricas en diferentes partes del mundo, 
abriendo oportunidades y generando amenazas a las PYMEs, que se ven presionadas a cambiar sus paradigmas ${ }^{4}$ gerenciales, y requieren rediseñar mecanismos de interrelación con el nuevo entorno. Entonces, la asociatividad surge como un mecanismo de cooperación entre pequeñas y medianas empresas que enfrentan la globalización de la economía.

La asociatividad permite resolver problemas conjuntos manteniendo la autonomía gerencial de las empresas participantes y los resultados logrados por los esfuerzos asociados, cualesquiera que sean los beneficios obtenidos, son del interés exclusivo de cada empresa. Esto constituye un verdadero estímulo al desarrollo de la asociatividad.

Son muchas las actividades que puede abarcar la cooperación. La asociatividad se puede establecer para múltiples propósitos desde el financiamiento hasta la investigación conjunta de determinado problema, y al mismo tiempo, abarcar las diferentes etapas de los procesos básicos de las empresas, diseño, manufactura, comercialización, servicio post-venta, etc. En principio no hay limitación del ámbito de la cooperación en la asociatividad, como tampoco hay restricciones para la participación respecto al tipo de actividad que desempeña la empresa participante.

Las características de la asociatividad otorgan a este mecanismo una alta flexibilidad de afiliación, operación y ámbito de acción que puede ser empleado por empresas insertadas en redes verticales u horizontales, e incluso por las que no pertenecen a ninguna red. Este mecanismo es exclusivo de las PYMEs, pues las grandes empresas constituyen cooperación a través de alianzas estratégicas y aún cuando una misma empresa pueda mantener múltiples alianzas estratégicas, cada una tiene un acuerdo específico entre dos partes. Por tanto, no se da el carácter colectivo.

Dadas las amplias bondades que la asociatividad ofrece a las PYMEs, su viabilidad estará en dependencia del alto grado de madurez para insertarse en un nuevo estilo de cooperación entre empresas. El desarrollo de un comportamiento de cooperación entre los empresarios dará sustento a la competitividad sistémica, permitiendo al mismo tiempo el cambio en las PYMEs nicaragüenses del estilo de dirección rígido, mediante la identificación de las similitudes.

No obstante, el MIFIC, a través de la DGPYME, debe crear un entorno institucional que estimule y soporte la existencia de mecanismos de cooperación, para que no constituya una barrera que desestimule el desarrollo de la cooperación entre empresas. Además, las estructuras creadas para fomentar la relación entre las empresas, no han recibido el soporte suficiente para desempeñar un papel beligerante y sólido y la institución, como tal, no ha sido lo suficientemente beligerante y firme en sus acciones, como para obtener el máximo provecho.

Para un mayor éxito en la asociatividad, es necesario entender lo qué es y no confundirla con otras estrategias individuales y colectivas. La asociatividad tiende a confundirse con una asociación de empresas con problemas comunes, que se asocian para presionar ante otra empresa o instancia gubernamental para una solución reivindicativa, generalmente coyuntural. Por ejemplo, facilidades crediticias, descuentos en la compra de materia prima 
o cupos en la asignación de órdenes de compra o despacho de materiales. Otra confusión de término es considerarla como una cooperativa, con sus tradicionales mecanismos de asambleísmo para tomar decisiones y el carácter social de su razón de ser.

La construcción de confianza entre empresarios aparece como un elemento clave; la necesidad de contar con experiencias exitosas para demostración, es de máxima importancia, ya que es un ejemplo y estímulo a la creación de nuevas iniciativas; la evaluación rigurosa para seguir de cerca el resultado de la relación entre las empresas, es imprescindible para que estas experiencias se afiancen.

Finalmente, el universo de actores con los que la asociatividad puede materializarse es múltiple y variado. Puede darse con: el estado, las PYMEs, las universidades, la cooperación

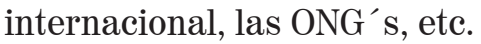

Las formas más conocidas de asociatividad son: núcleos y/o asociaciones empresariales, redes de servicios, cooperación empresarial como subcontratación o redes verticales, alianzas estratégicas o redes horizontales, distritos industriales o clusters y cadenas de valor.

\section{Desarrollo de Clusters competitivos}

Michael Porter ${ }^{5}$ define clusters como agrupamientos productivos o redes empresariales. Es decir, se trata de sistemas productivos constituidos por un número considerable de empresas independientes, predominantemente PYMEs, articuladas en cadenas productivas, concentradas geográficamente y especializadas sectorialmente.

Las PYMEs que operan en los distritos y clusters integran encadenamientos productivos, suelen complementarse y coordinarse entre sí, concentrándose y especializándose en distintas fases del proceso productivo. Los complejos productivos suelen extenderse verticalmente hacia canales de distribución y consumidores; lateralmente, hacia proveedores de productos o servicios complementarios; y hacia compañías relacionadas por los conocimientos, tecnologías o insumos comunes. Asimismo, los complejos productivos engloban a universidades, agencias que determinan estándares, institutos de capacitación profesional y asociaciones gremiales. Ser parte de un complejo productivo permite también a las compañías medirse y motivarse.

Para que las PYMEs rompan su aislamiento y se inserten en el mercado de productos y factores de la producción (insumos, tecnología, financiamiento, laboral, etc) es preciso articular los esfuerzos de un conjunto de empresas. Muchas veces esta articulación tiene que ser respaldada por entidades especializadas que respondan a los intereses de las PYMEs involucradas. Estos agentes desempeñan un rol importante para favorecer la colaboración entre empresas; revertir actitudes y conductas de los empresarios PYMEs que obstaculizan la asociatividad: individualismo, cortoplacismo, desconfianza, temor al oportunismo, falta de visión estratégica, resistencia al cambio, etc.; y minimizar y absorber los costos de transacción que dificultan la coordinación entre empresas (falta de información, dispersión territorial, empresariado sobreocupado, etc). 
La experiencia internacional de muchos distritos y clusters es contundente: La gente vive en la comunidad cerca del lugar de trabajo, gasta el dinero en el mismo lugar, contribuye al progreso de su localidad y contribuye a la acumulación de conocimientos y habilidades que se difunden en la comunidad. La disponibilidad de estos conocimientos y habilidades determina el atractivo de la comunidad como sistema productivo para generar nuevas iniciativas, atraer inversiones y reducir barreras a la entrada para nuevas PYMEs. Se crean empleos e ingresos y se genera así un círculo que causa prosperidad, confianza y autonomía.

Las PYMEs nicaragüenses requieren desarrollar su capacidad empresarial creando vínculos de apoyo que no pueden ser desarrollados mientras persista la competitividad negativa de "hacer campaña sucia para sacarte del mercado"; la información sea "juego mitológico de aspirantes a dioses"; y la desconfianza y la mentalidad de autosuficiencia competitiva permanezcan.

\section{Las alianzas estratégicas y el acceso a nuevos mercados}

La integración de las empresas es uno de los fenómenos cuyo origen es la globalización. Hay muchos ejemplos de empresas y alianzas que se han desarrollado con éxito en mercados internacionales. La política exterior de un país como Nicaragua, con profundas limitaciones económicas y políticas, debe ser un tema de primer orden a considerar.

Los nuevos escenarios plantean a las PYMEs el desafío de la internacionalización y la competitividad. Una debilidad tradicional de las PYMEs nicaragüenses es su tendencia a operar en forma aislada y particular. El paradigma del "yo empresario", "mi empresa”, "mi utilidad", impone barreras en la búsqueda de nuevas opciones estratégicas empresariales, limitando a los empresarios a asumir nuevos retos y desafíos de carácter internacional. El temor a fracasar ante la internacionalización y formación de alianzas estratégicas, hace que muchas PYMEs prefieran sobrevivir mientras se pueda y renacer con otra razón social.

La falta de experiencia en la negociación formal (legal) de una alianza estratégica, y la escasa información que brindan las empresas que promueven estas alianzas, es otro obstáculo al que tienen que hacer frente las PYMEs. Asimismo, el idioma y los contactos internacionales y la incipiente utilización de medios electrónicos de comunicación. Las PYMEs tienen poca presencia en Internet y en el comercio electrónico.

Por el contrario, las experiencias exitosas observadas internacionalmente evidencian la contribución de distintas fórmulas de cooperación al desarrollo competitivo y al proceso de internacionalización, y cómo en las uniones y alianzas cada parte pone algo: los socios extranjeros aportan la imagen y la calidad aprobada que envuelve a la sociedad establecida, mientras los nicaragüenses brindan un lugar desde donde expandirse por medio del $\mathrm{ALCA}^{6}$.

El rasgo distintivo de estos acuerdos es que promueven negocios que darán frutos en el mediano y largo plazo, pero requieren de grandes esfuerzos y sobre todo persistencia una vez iniciado el proceso de conquista de nuevos mercados. Aunque las alianzas estratégicas 
pueden ser peligrosas por la mezcla de culturas organizacionales y la dificultad para establecer y medir metas de desempeño.

En concreto, los acuerdos pueden abarcar compra o venta de productos y servicios, representaciones, distribución, compra o venta de tecnología, licencias de patente y marca, acuerdos de subcontratación o complementación productiva, franquicias, desarrollo de proyectos, compra o venta de participación accionaria e inversiones conjuntas.

Las PYMEs tienen mayor significación como exportadores indirectos que como exportadores directos, individuales o asociados. Tras la reorganización siguen los esfuerzos dirigidos a encontrar localizaciones más favorables para las empresas orientadas a la exportación, por ejemplo en la cercanía de puertos, y dirigidos también a mejorar la calidad; se ponen en marcha procesos de búsqueda para concentrar recursos en productos con muy buenas posibilidades de exportación. Siendo favorables las políticas para crear el entorno, surgen también nuevas PYMEs que, a veces, se orientan desde el principio a la exportación.

Existen distintas modalidades de inserción en el mercado internacional, que pueden potenciarse en el marco de un proceso de integración:

a) Exportación de un producto específico aprovechando un nicho de mercado.

b) Asociación con otras empresas para lograr el nivel de oferta adecuado que concrete negocios de exportación.

c) Exportación indirecta sea como proveedora de insumos para empresas con actividad exportadora, ya sea en un tramo inicial y/o intermedio de un proceso productivo cuya etapa final lo transforme en un producto exportable.

d) Alianzas estratégicas con firmas extranjeras.

Las posibilidades de que las PYMEs aprovechen las oportunidades de los procesos de integración, el ALCA o cualquier otro, radicará en el empleo de estrategias de identificación y aprovechamiento de nichos de mercado, pero teniendo en cuenta las prácticas empresariales de cooperación, mediante asociaciones de tipo horizontal con otras PYMEs, o a través de articulaciones con grandes empresas exportadoras.

\section{Notas}

1 Legalmente constituidas y registradas.

2 PROMICRO significa: Fortalecimiento y Promoción de las Organizaciones Gremiales, las Innovaciones Productivas y los Programas de Apoyo a las Microempresas en América Central. El proyecto PROMICRO es una iniciativa de la Organización Internacional del Trabajo (OIT).

3 Ramón Rosales. La asociatividad como estrategia de fortalecimiento de las PYMEs, en Sela, julio - septiembre 1997.

4 Paradigma; (del latín Paradigma) Cada uno de los esquemas formales a que se ajustan las palabras nominales y verbales para sus respectivas flexiones. Diccionario de la Real Academia Española. Edición 1992.

Dentro de este contexto se define paradigma como el conjunto de reglas que definen los límites y las posibilidades de éxito dentro de un determinado sistema. Las Pymes Latinoamericanas. Néstor P. Braidot y Eduardo Soto, noviembre 1999.

5 Michael E. Porter, Seminario "Competitividad en Centroamérica", San José, Costa Rica, Noviembre de 1995.

6 Área de Libre Comercio de las Américas. Proceso iniciado en la Cumbre de las Américas en 1994 para integrar las economías del Hemisferio Occidental en un solo acuerdo de libre comercio. 


\section{Referencias bibliográficas}

-BERRY, A., (sf). Las Tareas de la Pequeña y Mediana Empresa en América Latina -BRAIDOT, N. P., SOTO, E., (2001). Las PYMEs Latinoamericanas. Ed. IFEMA -CEPAL, (1998). Las PYME en Centroamérica y su Vinculación con el Sector Externo -DE OBESO, M., (1999). Estrategias Empresariales de las PYMEs Industriales Españolas

-ECHEVERRÍA, O. U., (1999).El Mayor Problema de las PYMEs: Su Propio Tamaño, Revista "Cuba: Investigación Económica" año 5, $\mathrm{N}^{0}$ 2, Ed. Instituto Nacional de Investigaciones Económicas

-FORO BOLÍVAR DE LA EMPRESA LATINOAMERICANA, (1997). Programa Bolívar, Caracas, Venezuela, pág. 127-137

-GARCÍA C., A., (1998). Globalización y Estrategias Alternativas para PYMEs

-GTZ, (1999). La Promoción a la Micro, Pequeña y Mediana Empresa en América Central

-INPYME, (2001). Plan Estratégico, Ed. Instituto Nicaragüense de Apoyo a la Pequeña y Mediana Empresa INPYME, Managua

-INPYME, (2001). Políticas de Fomento a las PYMEs, Ed. Dirección de Políticas de Fomento a las PYMEs Ministerio de Fomento, Industria y Comercio, Managua, -INPYME, (2001). Propuesta de Desarrollo para la Micro, Pequeña y Mediana Empresa Nicaragüense, Managua

-MARTÍNEZ, S., (1998). El Rol de la Micro y Pequeña Empresa en el Proceso de Apertura de Centro América, San José, Tesis presentada para optar al título de Doctor en Ciencia Económicas y Empresariales.

-PORTER, M. E., (2000). Estrategia Competitiva, Vigésima séptima reimpresión. México -PROYECTO PROMICRO (RLA/90/MO1/NET) DE LA ORGANIZACIÓN DEL TRABAJO (OIT), Oficina para América Central y Panamá, (1999). Propuesta de Desarrollo para la Microempresa Salvadoreña al año 1999, San Salvador

-Sistema de Información sobre la Microempresa en América Central (SIPROMICRO), Foro y discusión, Microempresa y Globalización. Febrero 2001. 